\title{
BMJ Open Quality Development of a hub and spoke model for quality improvement in rural and urban healthcare settings in India: a pilot study
}

\author{
Sushil Srivastava, ${ }^{1}$ Vikram Datta, ${ }^{2}$ Rahul Garde, ${ }^{3}$ Mahtab Singh, ${ }^{3}$ \\ Ankur Sooden (1D , ${ }^{3}$ Harish Pemde, ${ }^{4}$ Manish Jain, ${ }^{5}$ Poonam Shivkumar, ${ }^{6}$ \\ Akash Bang, ${ }^{5}$ Prabha Kumari, ${ }^{7}$ Sonia Makhija, ${ }^{8}$ Tarun Ravi, ${ }^{9}$ Sumita Mehta, ${ }^{10}$ \\ Bishan Singh Garg, ${ }^{11}$ Rajesh Mehta ${ }^{12}$
}

To cite: Srivastava S, Datta V, Garde R, et al. Development of a hub and spoke model for quality improvement in rural and urban healthcare settings in India: a pilot study. BMJ Open Quality 2020;9:e000908. doi:10.1136/ bmjoq-2019-000908

- Additional material is published online only. To view please visit the journal online (http://dx.doi.org/10.1136/ bmjoq-2019-000908).

SS and VD contributed equally.

Received 26 December 2019 Revised 9 June 2020 Accepted 7 July 2020

Check for updates

(c) Author(s) (or their employer(s)) 2020. Re-use permitted under CC BY-NC. No commercial re-use. See rights and permissions. Published by BMJ.

For numbered affiliations see end of article.

Correspondence to

Prof. Vikram Datta;

drvikramdatta@gmail.com

\section{ABSTRACT}

Objective Hub and spoke model has been used across industries to augment peripheral services by centralising key resources. This exercise evaluated the feasibility of whether such a model can be developed and implemented for quality improvement across rural and urban settings in India with support from a network for quality improvement. Methods This model was implemented using support from the state and district administration. Medical colleges were designated as hubs and the secondary and primary care facilities as spokes. Training in quality improvement (QI) was done using WHO's point of care quality improvement methodology. Identified personnel from hubs were also trained as mentors. Both network mentors (from QI network) and hub-mentors (from medical colleges) undertook mentoring visits to their allotted facilities. Each of the participating facility completed their QI projects with support from mentors.

Results Two QI training workshops and two experience sharing sessions were conducted for implementing the model. A total of 34 mentoring visits were undertaken by network mentors instead of planned 14 visits and rural hub-mentors could undertake only four visits against planned 18 visits. Ten QI projects were successfully completed by teams, $80 \%$ of these projects started during the initial intensive phase of mentoring. The projects ranged from 3 to 10 months with median duration being 5 months.

Discussion Various components of a health system must work in synergy to sustain improvements in quality of care. Quality networks and collaboratives can play a significant role in creating this synergy. Active participation of district and state administration is a critical factor to produce a culture of quality in the health system.

\section{INTRODUCTION}

More than 8 million people die each year in low-income and middle-income countries (LMICs) due to conditions easily amenable to treatment. Poor quality care is one of the major contributing factors to this number. Although there has been an increased utilisation of the health services in most of the
LMICs, it has been marred by suboptimal quality of services. ${ }^{1}$ Efforts to introduce and scale up quality improvement (QI) interventions in LMICs have met severe bottlenecks leading to inadequate diffusion of QI across the health systems. ${ }^{1}$

Providing quality care is a way to provide universal health coverage in LMICs. In order to absorb and embed quality practices into their health systems, LMICs need to be supported and guided to help spread the QI methods. Implementing quality of care requires investment of resources. Often this investment is not beyond reach of even the poorest countries. ${ }^{2}$ Absence of a quality of care culture in existing policies has made most countries approach QI as project-based activity and not as an essential component of the health system. ${ }^{3}$ These policies also affect the existing linkages between the various levels of health system, namely, micro-level (eg, at health facility level), meso-level (eg, at district level, QI network/collaboratives) or the macro-level (eg, at state or national level), and have an overarching impact on the outcomes of care.

The hub and spoke model (HSM) has been used for augmenting weak peripheral settings by connecting them to a resource replete hub. ${ }^{4}$ Such a model could be a natural fit in resource poor health systems, commonly encountered in LMICs. The resource rich hub can support a peripheral facility by material means and in terms of offering its technical expertise and trained human resource. The effectiveness of the linkage is dependent on multitude of factors, key factor being organisational culture at both the hub and spoke. The current paper is an attempt to describe the process of setting up of an operational HSM across rural and urban settings in 
India. To the best of our knowledge, there is no published literature on the use of this methodology for scaling up of QI initiatives across rural and urban settings in LMICs. The study was funded by WHO's South-East Asia Region Office (WHO-SEARO) and implementation of QI was done by Nationwide Quality of Care Network, India. ${ }^{5}$ The learnings and major challenges encountered in this process are presented in this paper.

\section{METHODS}

The QI network catalysed the implementation of the HSM of QI to build a sustainable QI linkage between health facilities. The implementation started from June 2018 to May 2019 at the Wardha District in Maharashtra state (rural setting) and in North and North West districts of Delhi state (urban setting).

Aim of this work was to establish QI mentoring linkage between tertiary care (hub) and secondary/primary care health facilities (spokes) in a rural and urban districts of India in maternal and newborn care settings, over the course of 1 year. For this aim, the process measure was the number of mentoring visits received by each facility from a mentor, and the outcome measure was the percentage of facilities that successfully completed their QI projects.

The model consisted of medical college hospitals designated as 'HUB' and the district hospitals (DHs), a rural hospital and a primary health centre were designated as 'SPOKE' facilities. The facilities with high delivery load ${ }^{6}$ and good access were selected to be part of this exercise. They were selected in consultation with state and district health officials of National Health Mission (NHM) (see table 1 for details). These facilities catered to about $60 \%$ of rural district's delivery load and about $20 \%$ of urban district's delivery load. Prior to the initiation of the implementation, formal consent and approvals were obtained from the concerned district authorities, administrative and clinical heads of the participating sites. Point of care quality improvement (POCQI) workshops were conducted for maternal and newborn healthcare providers from the hub and spoke facilities. POCQI ${ }^{7}$ is a simple four-step method of identifying, prioritising and solving problems by iterative learning using the Plan-Do-Study-Act cycles. The identified doctors and nurses from hub-facility were subsequently coached as hub-mentors. For the purpose of this implementation exercise, the participating hub facilities were coded as rural medical college and urban medical college. The spoke facilities from rural district were coded as R1, R2 and R3 and from urban districts as U1 and U2. Details about these facilities and the districts is described in table 1.

After POCQI training, teams from hub and spoke facilities worked on their identified problems. They were guided by mentors (network and hub based) using both onsite mentoring visits and telephone/WhatsApp. Mentoring visits by network mentors were planned from second month of the implementation with hub-mentors undertaking their visits after 6 months of implementation. A total of two visits per facility were planned for rural and urban districts by network mentors (ie, total of 14 visits). The hub-mentors were scheduled to undertake one visit per facility per month in their respective districts (ie, a total of 30 visits). The implementation plan had a flexibility to modify the number of onsite mentoring visits within the budgetary constrained as per the felt need. Besides mentoring visits, two experience sharing cum learning sessions were planned, one each, at rural and urban districts for teams to share their results and learnings.

The implementation plan was developed using the 'Point of Care Quality Improvement - Program Management Guide' published by WHO-SEARO ${ }^{8}$ and after consulting the state and district health officials of Delhi and Maharashtra state. Components of this plan are shown in table 2 . The linkages between the facilities and various stakeholders involved are shown graphically in figure $1 \mathrm{~A}$. figure $1 \mathrm{~B}$ and $\mathrm{C}$ shows what we were able to achieve vis a vis the planned linkage between health facilities (Figure 1A-C).

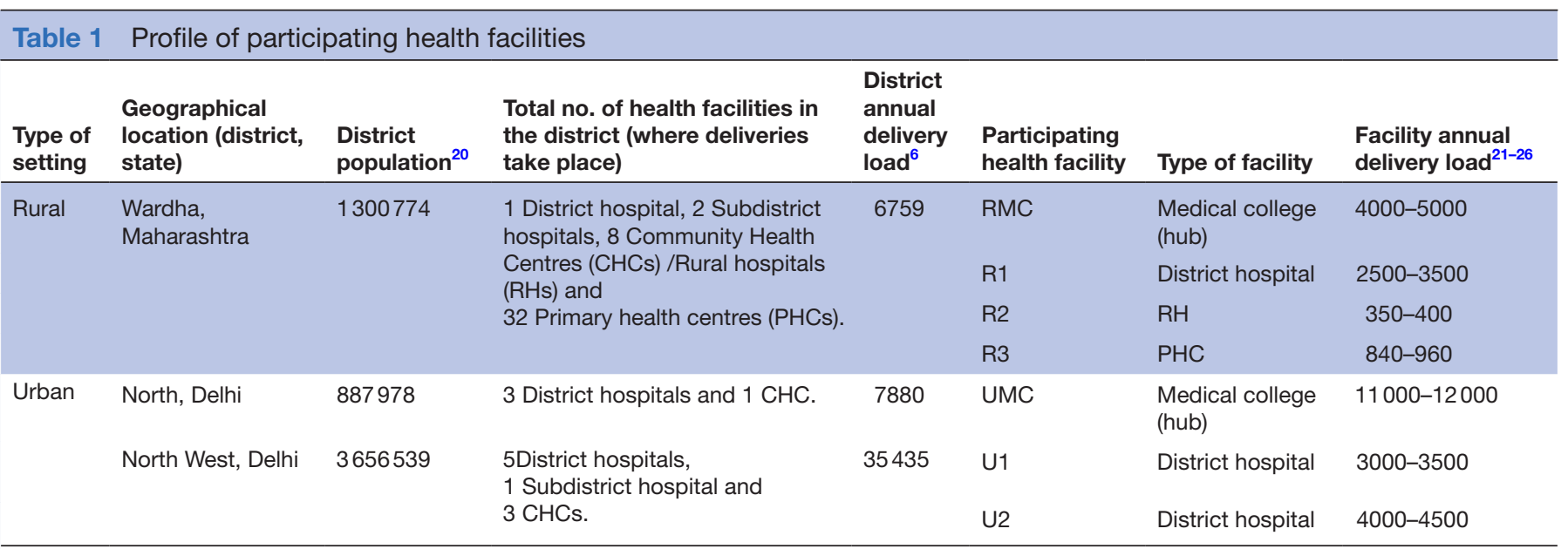

RMC, rural medical college; UMC, urban medical college. 
Table 2 Components of the district level quality improvement programme (hub-and-spoke) in project districts of Maharashtra (Wardha) and Delhi (North/North West)

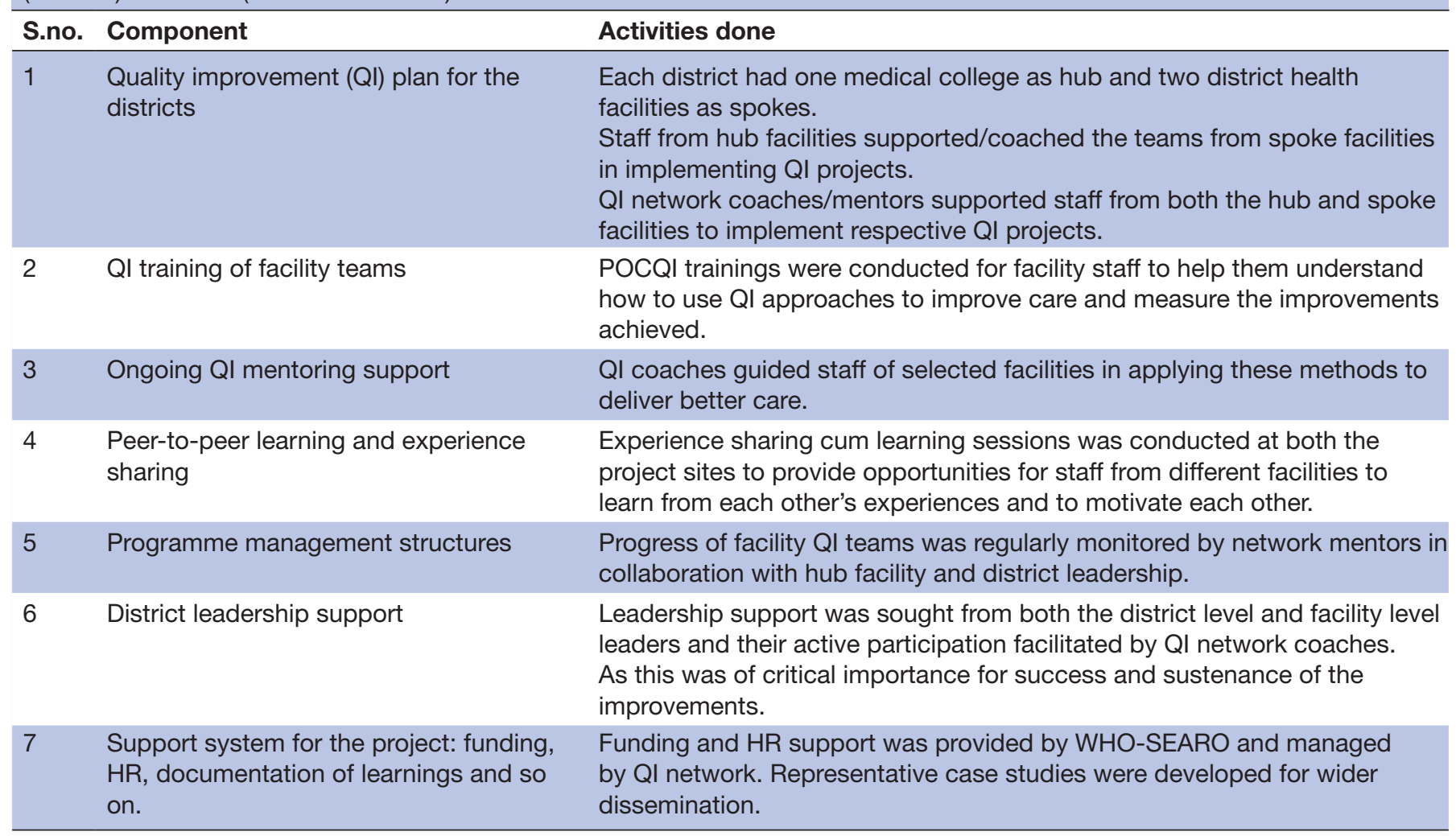

POCQI, point of care quality improvement.

\section{Patient involvement}

No patients were involved in this study because the main focus of the study was to identify factors affecting the implementation of QI initiatives in rural and urban settings. Likewise, no patients were involved in the development of the research questions, outcome measures, recruitment and the conduct of the study. The results were disseminated through experience sharing workshops to facility teams, funding partner and governmental agencies.

A total of 10 QI projects were completed during the study using the POCQI methodology. Of these five were completed by the rural hub, three by rural spokes and two by urban spoke facilities. Majority of facilities could only complete one QI project during the 1-year duration. Data and indicators were collated and analysed by the participating facilities with the active support of both the network and hub-mentors. The data were manually accessed and recorded from field registers and patient records by the respective QI teams. The data thus captured were analysed using Microsoft Excel 2016 and represented using time series charts to make it easy for spoke facilities to interpret the data. Kindly refer to table 3 for results and supplement for time-series graphs.

\section{RESULTS}

The results achieved by the QI teams from rural and urban facilities and our observations regarding the implementation of hub-and-spoke model are presented here. The results are presented under two sections: (A) hub and spoke implementation model for QI and (B) QI projects at individual facilities.

1. Hub and spoke implementation model for QI: a total of 34 mentoring visits were undertaken by the network mentor, against planned 14 visits, that is, an increase of $242 \%$. In comparison, rural hub-mentors could undertake only four visits against planned 18 visits, that is, only $22 \%$ visits could be completed. Mentors from urban-hub could not complete any mentoring visits. Detailed schematic of all the activities undertaken for the HSM is shown in the figure 2A. Progress of process and outcome indicators of the HSM over the 1year implementation period are shown in figure 2B. As can be seen in the figure, the mentoring visits started after a gap of 2 months and 6 months in rural and urban facilities, respectively. In the rural facilities, $50 \%$ of total mentoring visits were completed in the initial 3 months of starting the mentoring, which led to 8 out of $10(80 \%)$ QI projects getting initiated during this period. In the second half of the year, rest of the mentoring visits were completed and two more QI projects were initiated. Two experience sharing meeting were conducted for rural and urban facilities. The results of individual facilities are presented below.

2. QI projects at individual facilities: the results of QI projects by facility teams are shown in table 3 . As can be seen from the table, all QI teams chose independent 

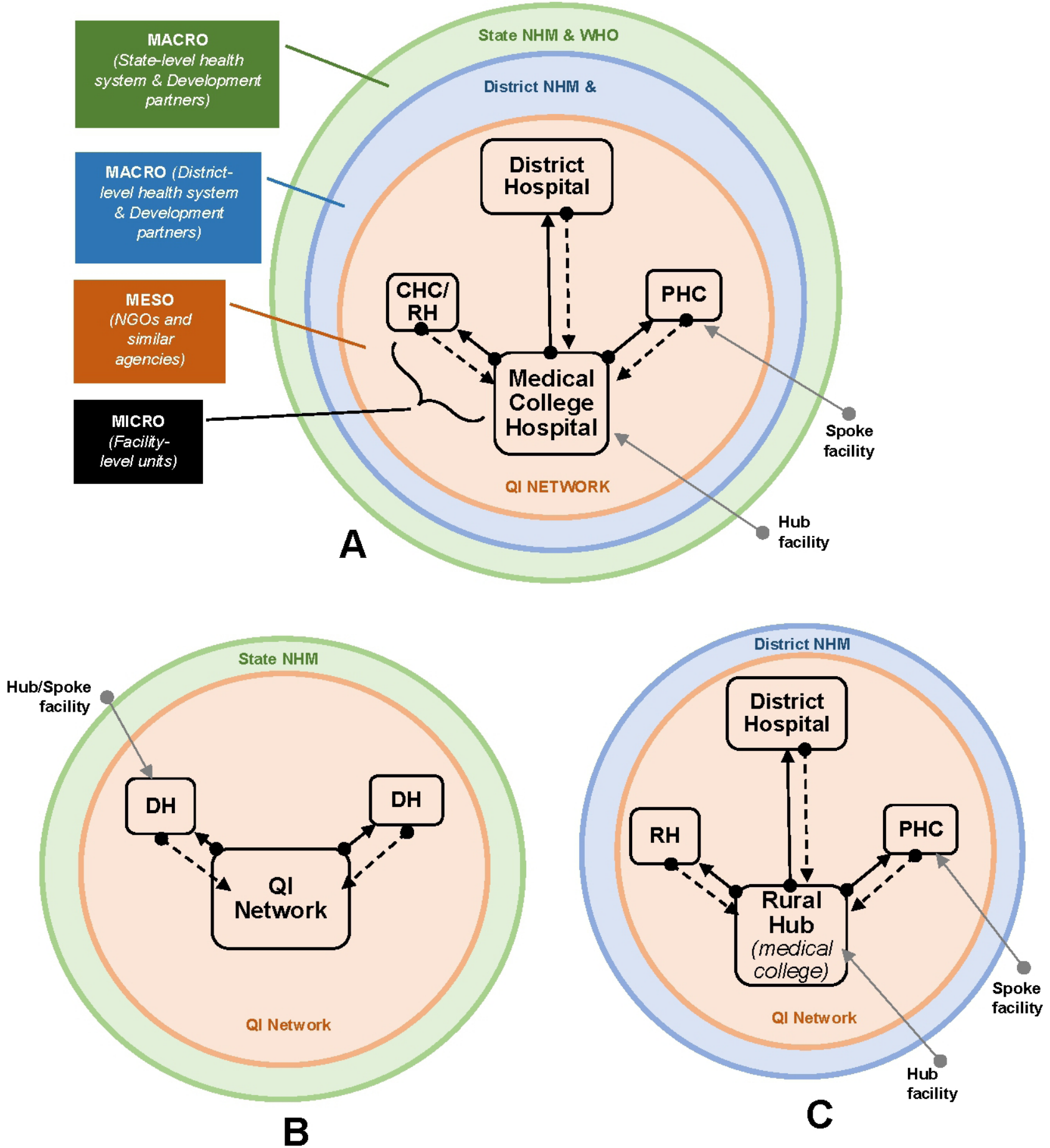

Figure 1 Graphical representation of (A) planned district hub-and-spoke model for quality improvement (QI). (B) Implemented model in urban setting. QI network became the 'hub' facility for spokes (ie, DHs). (C) Implemented model in rural setting. Solid arrows highlight the mentoring support that medical colleges provided and dashed arrows show the direction of data flow pertaining to Ql projects.CHC, community health centre; DH, district Hospital; NHM, National Health Mission; NGO, non governmental organisation; $\mathrm{PHC}$, primary health centre; $\mathrm{RH}$, rural hospital.

projects. The projects ranged from 3 months to 10 months, with median duration of 5 months. Urban hub facility was unable to undertake any QI project. A detailed schematic of all the activities undertaken for the HSM is shown in figure $2 \mathrm{~A}$, which shows the temporal pattern of each of the QI projects (figure 2A,B).

\section{DISCUSSION}

HSM was initially developed in the aviation and logistics industry for efficient and effective use of resources. ${ }^{9-11}$ Implementation of this model helped the organisations to improve their productivity, which ultimately led to its adoption by various industries across the world. ${ }^{910}$ Conceptually, 


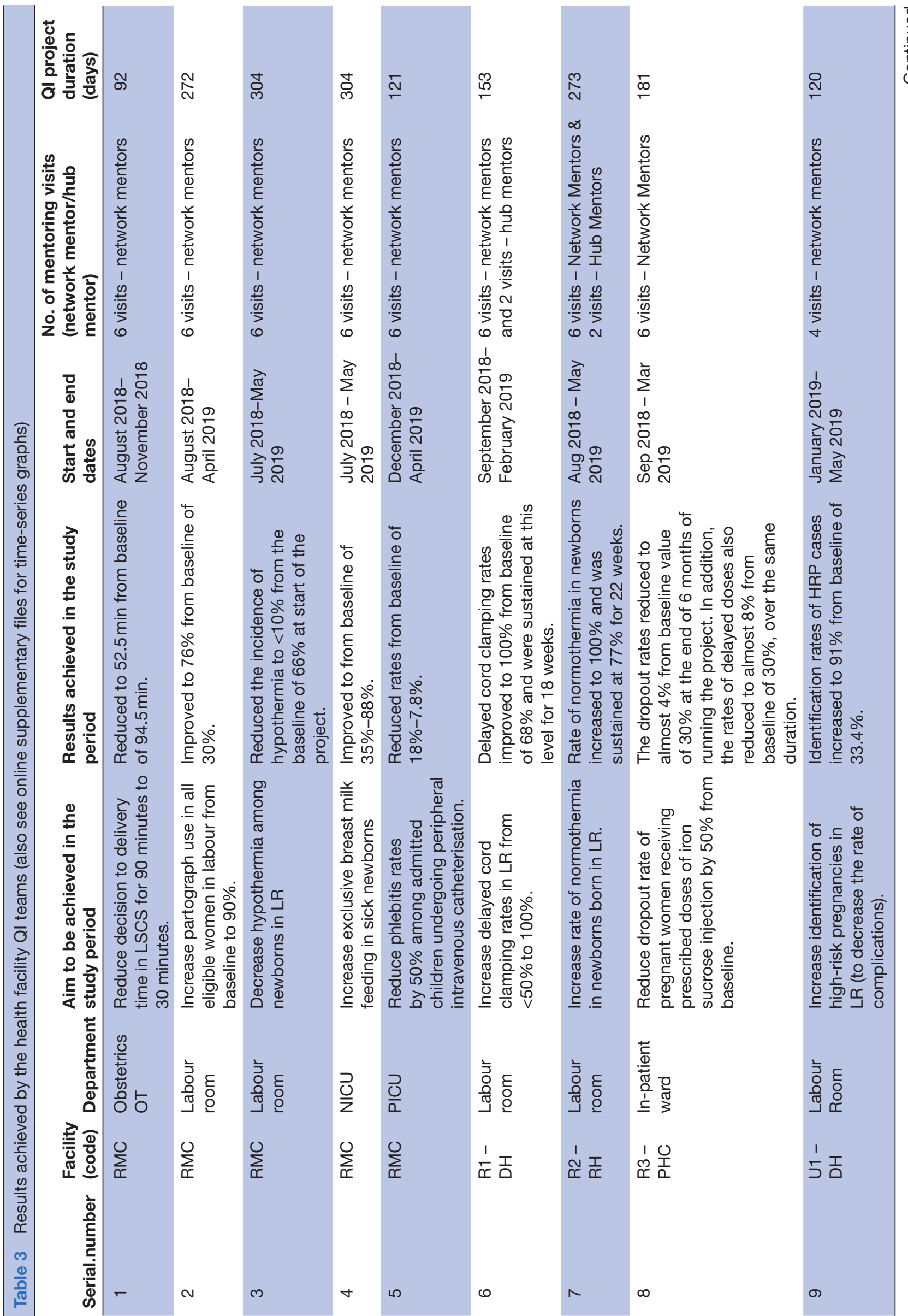


HSM is a natural fit for healthcare service delivery, especially, in resource-limited settings where it can help to augment the deficiencies in the peripheral facilities by linking them to a resource replete central hub facility. Use of this design for building QI skills is a novel innovation.

We tested the district level implementation model for scale up of QI using the hub and spoke mechanism for maternal and newborn care settings. This was adapted contextually for rural and urban districts across India. This was the first attempt at developing a model for upscaling QI at district level; it helped us to understand various factors that affect uptake of QI skills at different levels. It made us aware of the limitations of the model in implementation of QI at scale.

A key objective of this work was development and demonstration of a sustainable QI handholding/ supportive supervision model. District and State Health officials, health facility staff and network mentors (from QI network) were the key participants in this process. The purpose of developing these linkages between the facilities in a district was to foster a culture of QI in the local health system. This is an important step in the direction of developing a health system that can deliver universal health coverage for all, as mentioned in the Lancet commission report on high-quality health systems. ${ }^{1}$ The report had highlighted that globally majority of initial attempts at QI are at facility level (micro-level). Such efforts are often not sustainable and may not lead to a system-wide improvement.

The meso-level efforts (eg, a QI network or collaborative), can be a catalyst for increasing uptake and spread of QI across facilities as demonstrated in multiple studies. ${ }^{12-15}$ However, interventions directed at mesolevel are need to be operationalised more often in health systems. ${ }^{1} 1617$ Sustenance of micro-level interventions without meso-level support is a serious bottleneck across all health systems. ${ }^{1}$ This finding from the commission is in conformity with our observations, as the 'hub' facilities in both rural and urban areas could not handhold 'spoke' facilities effectively for implementing QI skills. To sustain the improvements in the hub and spoke facilities, extensive mentoring support from the network QI mentors was required. This reiterates the fact that microlevel interventions require extensive meso-level support for medium term sustenance.

It was seen that the district and state level administrative buy-in/ownership was deficient. This was possibly due to lack of insight on benefits of implementation of QI at scale. ${ }^{18}$ This became one of the major bottlenecks in the effective implementation of the HSM. The major learning from this implementation exercise was that in absence of effective handholding and support at macrolevel long-term sustenance of any micro-level and mesolevel interlinked process is a formidable challenge.

Recognising these challenges, India has embarked on a mission to provide universal health coverage for all its citizens through Ayushman Bharat Scheme and the LaQshya initiative for improving quality of maternity care. ${ }^{19}$ The 


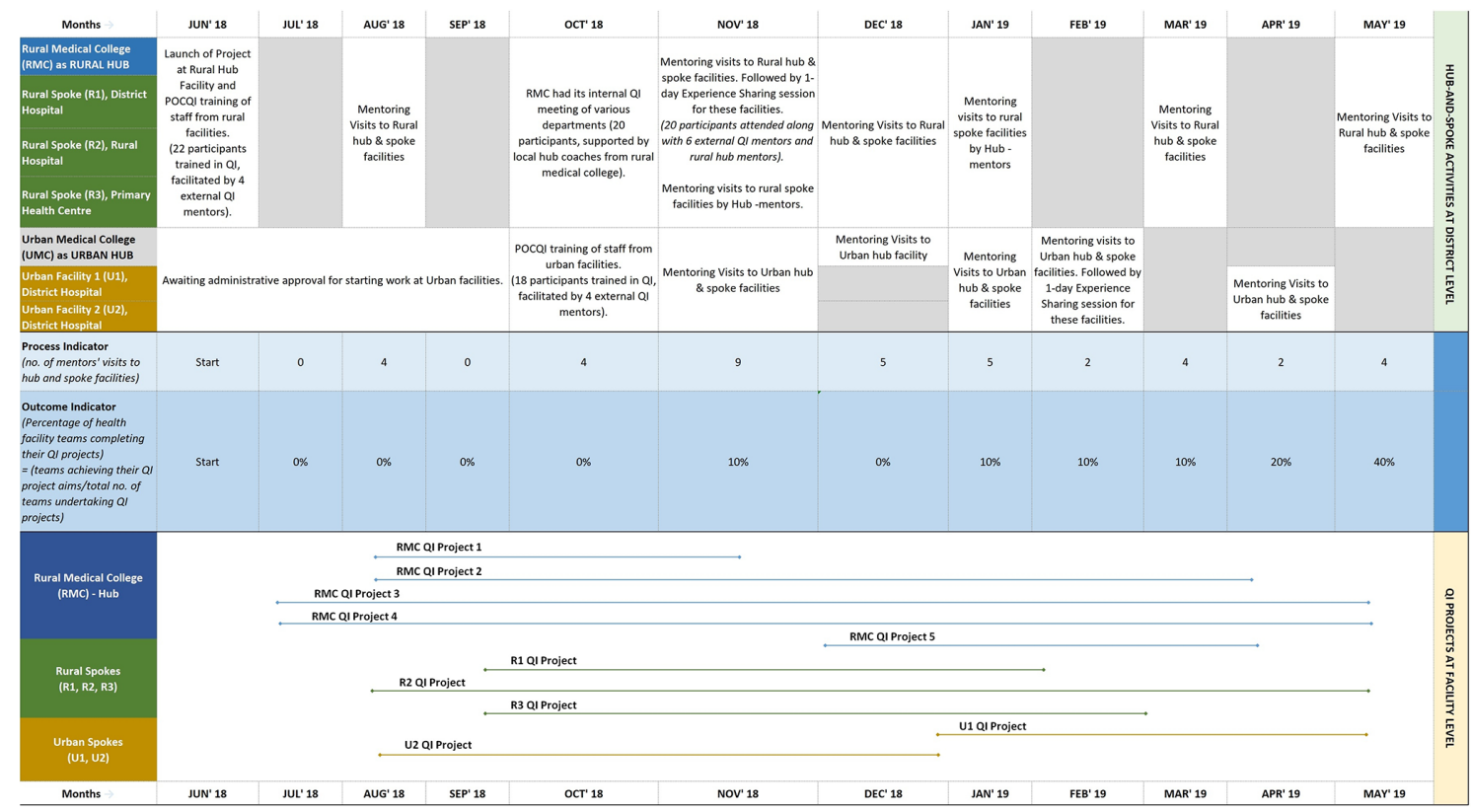

\section{A}

Progression of process and outcome indicators for hub-and-spoke work for facility QI linkages in two districts of India

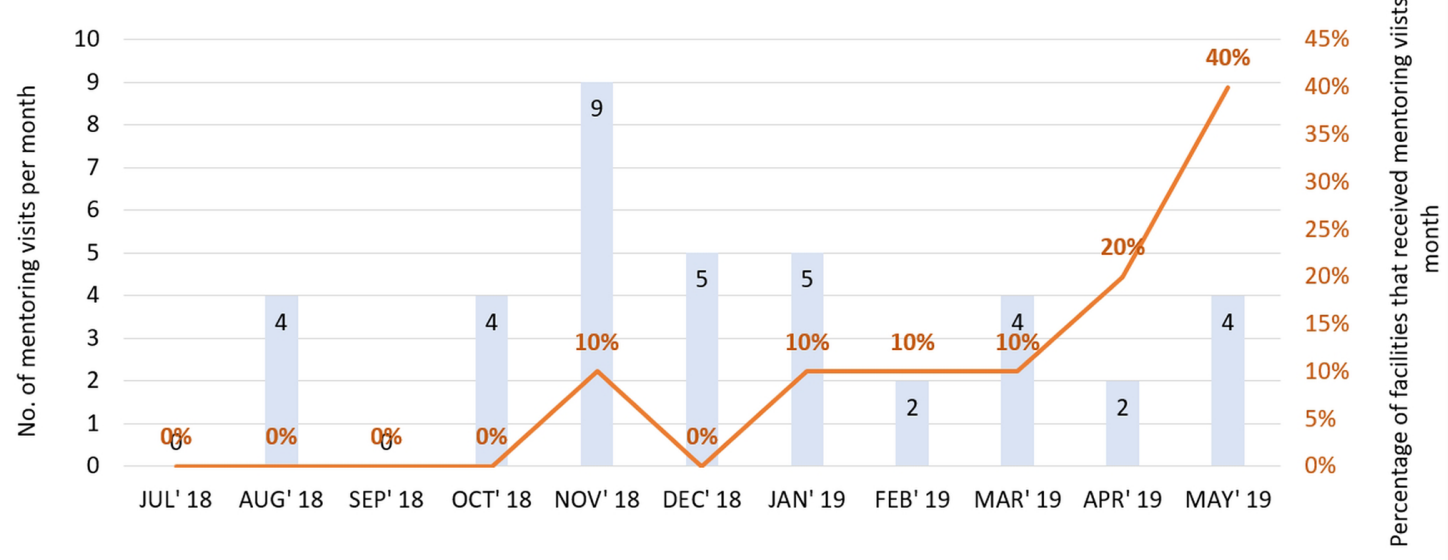
B

Process Indicator
(no. of mentors' visits to hub and spoke facilities) Start $\quad$ Outcome Indicator
(Percentage of health facility teams completing their QI projects) Start

Figure 2 (A) Schematic of hub and spoke model in rural and urban districts. (B) Progression of process and outcome indicators of the hub and spoke work done in rural and urban districts.

provisions of the aforementioned schemes have catalysed the process of onsite mentoring and handholding of medical colleges with an aim to develop them as regional resource centres for QI. This has been carried out with an objective of developing an operational HSM at the national level. Some of the QI team members and mentors from this implementation exercise are now part of the National Mentoring Group notified by the Ministry of Health \& Family Welfare, Government of India. This process is fostering a QI culture in the health system with full support from macro-level and active involvement of micro-level and meso-level facilities. Development of national mentoring process is a conscious exercise to overcome challenges and build on the learnings of the HSM.
The main learnings from this exercise were: (1) this model helped in sensitisation of teams to the POCQI methodology and helped them to test and use the methodology in field conditions with ease; (2) for effective uptake and successful implementation of QI, initial intensive onsite mentoring is an essential requirement; and (3) absence of effective macro-level support is the major roadblock for effective implementation of any HSM.

Challenges experienced while operationalising the model are grouped for ease of understanding into microlevel meso-level and macro-level.

- At micro-level: absence of an enabling environment at facility level created a culture that gave little incentive for health facility staff to learn QI skills. It was 


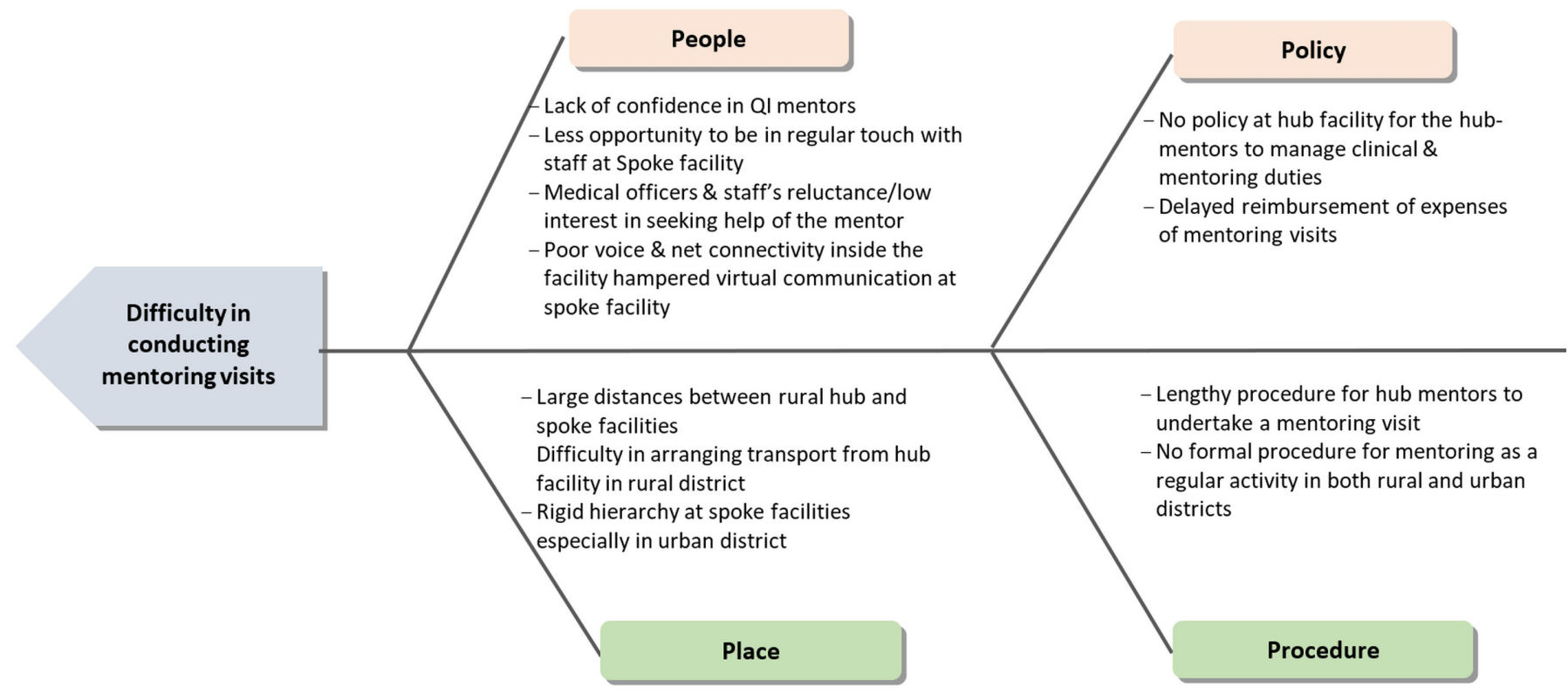

Figure 3 Fishbone diagram showing challenges faced by hub-based mentors in conducting mentoring visits to spoke facilities. QI, quality improvement.

further compounded by a rigid hierarchical system and partial administrative support especially encountered in the urban hub facility. Maintaining motivation in physicians was a challenge. Both the rural and urban facilities lacked dedicated hospital QI personnel, especially at the urban-hub facility; this made healthcare providers visualise QI as extra work, which distracted them from their regular patient care. Non-provision of transportation for hub mentors to their allocated spoke facilities added to poor motivation to undertake mentoring visits. See figure 3 for fishbone diagram of factors that hindered mentoring visits by hub-mentors.

- Meso-level: budgetary constraints for conducting intensive onsite mentoring led to visits being conducted by one out of the two network mentors. Delay in approvals for starting activities in urban setting led to inability of urban hub to become functional in sync with project timelines. Lack of sensitisation about QI methodology among the district and NHM officials led to deficient buy-in/ownership and consequent support. Lack of active participation from the district officials and state NHM officials on account of other competing priorities for district and state health officials.

- Macro-level: lack of ownership and financial provisioning by the state administration for scaling up of this initiative across districts led to non-sustenance of the operational model. Irregular monitoring and evaluation of the QI work progress by the state and district officials led to lack of accountability for the process. The transfer of a senior official from health department led to significant delays in operationalising of the project.

\section{Limitations of this work}

There were a few limitations in our work. The work was conducted in two districts, which may not represent the health system of a country as diverse as India. The major bottlenecks encountered in the implementation process may not necessarily operate in other similar settings. It only offers possible pointers to future efforts on implementing QI programmes at scale. Active community participation was deficient in the implementation model. As multiple facilities worked on diverse improvement aims without using a collaborative approach, a systemwide improvement could not be demonstrated in the short span of 1 year. Moreover, the short duration of this work, sustenance of hub and spoke learnings beyond the project duration could not be determined.

\section{CONCLUSION}

HSM as a concept helps in better utilisation of resources in a system. Multiple factors operating at macro-level, meso-level and micro-level interact in a complex manner to predicts if the model will be successfully implemented or not. Change in organisational culture is a slow process and an important factor for success of any such model. A strong political will, administrative support and empowered facility teams working in an environment of physical and psychological safety would go a long way in replicating HSM at scale. As this was an initial attempt to develop a HSM across rural and urban settings of India, further large-scale studies with community participation should be designed to overcome the challenges and limitations brought out from this work. If implemented at scale, the HSM has potential to revolutionise the culture of quality and patient safety across resource constrained LMIC settings. 
Author affiliations

${ }^{1}$ Department of Pediatrics, University College of Medical Sciences, Delhi, India

${ }^{2}$ Department of Neonatology, Lady Hardinge Medical College, New Delhi, India

${ }^{3}$ Nationwide Quality of Care Network, New Delhi, India

${ }^{4}$ Department of Pediatrics, Lady Hardinge Medical College, New Delhi, India

${ }^{5}$ Department of Pediatrics, Mahatma Gandhi Institute of Medical Sciences,

Sevagram, Wardha, India

${ }^{6}$ Department of Obstetrics \& Gynaecology, Mahatma Gandhi Institute of Medical

Sciences, Sevagram, Wardha, India

${ }^{7}$ Department of Obstetrics and Gynaecology, Bhagwan Mahavir Hospital, Delhi, India

${ }^{8}$ Department of Pediatrics, Bhagwan Mahavir Hospital, Delhi, India

${ }^{9}$ Department of Pediatrics, Babu Jagjivan Ram Memorial Hospital, Delhi, India

${ }^{10}$ Department of Obstetrics \& Gynaecology, Babu Jagjivan Ram Memorial Hospital,

Delhi, India

${ }^{11}$ Department of Community Medicine, Mahatma Gandhi Institute of Medical

Sciences, Sevagram, Wardha, India

${ }^{12}$ WHO SEARO, New Delhi, India

Twitter Ankur Sooden @AnkurSooden

Contributors VD and SS led the overall design of the research and provided leadership for the study. VD, MS, SS, RG and AS contributed to the design of the research, acquired and analysed the data. MJ, PS, AB, PK, SoM, TR, SuM and BSG led the individual improvement projects and helped in the interpretation of the data thereof. MS, SS, HP and VD were responsible for conducting onsite mentoring visit to the hub facilities. MJ and AB mentored the spoke facilities. VD, SS, RG, AS, MS and RM contributed to drafting of the manuscript; all authors revised it critically and approved the submission. VD, SS, RG, MS and AS have contributed in the revision of the manuscript. All authors have approved the final version.

Funding WHO-SEAR Office, New Delhi, funded the study.

Disclaimer The funding agency had no bearing on methodology, data collection and analysis and results of this study. The opinions expressed herein are those of the authors and do not represent the official views of the WHO.

Competing interests RM is employed with the WHO-SEAR Office. He was responsible for release of funds for this project.

Patient and public involvement Patients and/or the public were not involved in the design, or conduct, or reporting, or dissemination plans of this research.

Patient consent for publication Not required.

Provenance and peer review Not commissioned; externally peer reviewed.

Data availability statement Data are available on reasonable request. All data relevant to the study are included in the article or uploaded as supplementary information. All deidentified data are available with the corresponding author and may be shared on reasonable request.

Open access This is an open access article distributed in accordance with the Creative Commons Attribution Non Commercial (CC BY-NC 4.0) license, which permits others to distribute, remix, adapt, build upon this work non-commercially, and license their derivative works on different terms, provided the original work is properly cited, appropriate credit is given, any changes made indicated, and the use is non-commercial. See: http://creativecommons.org/licenses/by-nc/4.0/.

ORCID iD

Ankur Sooden http://orcid.org/0000-0002-8295-7978

\section{REFERENCES}

1 Kruk ME, Gage AD, Arsenault C, et al. High-Quality health systems in the sustainable development goals era: time for a revolution. Lancet Glob Health 2018;6:e1196-252.

2 World Health Organization, Organisation for Economic Co-operation and Development, and The World Bank. Delivering quality health services: a global imperative for universal health coverage, Licence: CC BY-NC-SA 3.0 IGO. Geneva: World Health Organization, Organisation for Economic Co-operation and Development, and The World Bank, 2018.
3 Leatherman S, Ferris TG, Berwick D, et al. The role of quality improvement in strengthening health systems in developing countries. Int J Qual Health Care 2010;22:237-43.

4 Elrod JK, Fortenberry JL. The hub-and-spoke organization design: an Avenue for serving patients well. BMC Health Serv Res 2017;17:457.

5 Datta V, Srivastava S, Singh M. Formation of quality of care network in India: challenges and way forward. Indian Pediatr 2018;55:824-7.

6 National Health Mission. Health management information system (HMIS) standard reports 2018-19. Ministry of health and family welfare, Govt. of India. Available: https://nrhm-mis.nic.in/ hmisreports/frmstandard reports.aspx

7 Deorari A, Mehta R, Livesley N. Improving the quality of care for mothers and newborns in health facilities: point of care quality improvement. coaching manual. New Delhi, India: World Health Organization, Regional Office for South-East Asia, 2019.

8 WHO SEARO. Programme management guide 2018. Available: http://www.pocqi.org/wp-content/uploads/2018/07/ProgrammeManagament-Guide.pdf [Accessed 24 Aug 2019].

9 Govindarajan V, Ramamurti R. Delivering World-Class health care, Affordably. Harvard business review, 2014. Available: https://hbr.org/ 2013/11/delivering-world-class-health-care-affordably [Accessed July 29, 2019].

10 Delve S. How the Hub-and-Spoke Model Transformed the Transportation Industry - Inbound Logistics, 2019. Available: https:// www.inboundlogistics.com/cms/article/how-the-hub-and-spokemode-transformed-the-transportation-industry [Accessed 5 Aug 2019].

11 Devarakonda S. Hub and spoke model: making rural healthcare in India affordable, available and accessible. Rural Remote Health 2016;16:1-8.

12 USAID-ASSIST. The improvement collaborative: an approach to rapidly improve health care and scale up quality services. June 2008 available at. Available: https://www.usaidassist.org/sites/assist/files/ the_improvement_collaborative.june08.pdf

13 Horbar JD, Plsek PE, Leahy K, et al. NIC/Q 2000: establishing habits for improvement in neonatal intensive care units. Pediatrics 2003;111:e397-410.

14 Lee HC, Kurtin PS, Wight NE, et al. A quality improvement project to increase breast milk use in very low birth weight infants. Pediatrics 2012;130:e1679-87

15 Dunbar AE, Sharek PJ, Mickas NA, et al. Implementation and case-study results of potentially better practices to improve pain management of neonates. Pediatrics 2006;118 Suppl 2:S87-94.

16 Bradley EH, Taylor LA, Cuellar CJ. Management matters: a leverage point for health systems strengthening in global health. Int $J$ Health Policy Manag 2015;4:411-5.

17 Lega F, Prenestini A, Spurgeon P. Is management essential to improving the performance and sustainability of health care systems and organizations? A systematic review and a roadmap for future studies. Value Health 2013;16:S46-51.

18 Mohanan M, Hay K, Mor N. Quality of health care in India: challenges, priorities, and the road ahead. Health Aff 2016;35:1753-8.

19 Gopal KM. Strategies for ensuring quality health care in India: experiences from the field. Indian J Community Med 2019;44:1-3.

20 Registrar General of India, Government of India, New Delhi. Census of India, 2011. Available: http://censusindia.gov.in/ [Accessed $16 \mathrm{Jul}$ 2019]

21 MGIMS, Sevagram, District Wardha, Maharashtra, India. Hospital in numbers (2011-2016). Available: https://www.mgims.ac.in/index.php/ hospital/hospital-in-numbers [Accessed 24 Jul 2019].

22 Medical Records Department, Rural Hospital. Hospital records. Pulgaon, District Wardha, Maharashtra, India, 2018.

23 Medical Records Department, PHC. Hospital records. Anji, District Wardha, Maharashtra, India, 2018.

24 Medical Records Department, Dr Baba Saheb Ambedkar Hospital, Delhi. Hospital Record of Indoor Services (Obstetrics \&Gynaecology). Available: http://164.100.72.17/wps/wcm/connect/doit dbsah/ DBSAH/Home/Departments/OBSTETRICS+AND+GYNECOLOGY/ INDOOR+SERVICES [Accessed 25 Jul 2019].

25 Medical Records Department, Dr. Baba Saheb Ambedkar Hospital, Delhi. Hospital clinical data. available from. Available: http://bsamch. ac.in/Misc/clinical.pdf [Accessed July 25, 2019].

26 Medical Records Department, BJRM Hospital, Delhi, India. Hospital records, 2018. unpublished data. 